\title{
Implantable Artificial Capillary Unit for Pancreatic Islet Allograft and Xenograft
}

\author{
W. J. Tze, F. C. Wong, and L. M. Chen \\ G. F. Strong Laboratory for Medical Research, Department of Paediatrics, University of British Columbia, \\ Vancouver, Canada
}

\begin{abstract}
Summary. Implantable artificial capillary units containing approximately 1,200 allogeneic rat islets, or approximately 3,000 xenogeneic rabbit or human islets as an implantable artificial endocrine pancreas (IAEP) were implanted in streptozotocin-induced $(55 \mathrm{mg} / \mathrm{kg}$ ) diabetic rats. A total of 26 rats received IAEP containing allogeneic islets. Twenty were short term experiments which lasted for $12-24 \mathrm{~h}$. Four recipients survived between 1-3 days and the remaining two for 4 and 11 days respectively. Five diabetic rats received IAEP containing xenogeneic islets. The four recipients of IAEP containing rabbit islets survived up to 4 days while the recipient of IAEP containing human islets survived for 8 days. Following implantation, a decrease of plasma glucose from the initial value of $500 \mathrm{mg} / \mathrm{dl}$ to normoglycaemia and a corresponding increase in circulating levels of insulin up to $100 \mu \mathrm{U} / \mathrm{ml}$ were observed in the recipient animals. Furthermore the IAEPs were shown to produce a near normal plasma glucose and insulin response to an intravenous glucose tolerance test. These findings suggest the feasibility of achieving amelioration of diabetes with allogeneic or xenogeneic pancreatic islets implanted as an artificial endocrine pancreas unit in the experimental animals which has the potential of future clinical application in man.
\end{abstract}

Key words: Artificial endocrine pancreas, capillary unit, allogeneic, xenogeneic, pancreatic islets, transplantation.

There is increasing interest in the transplantation of isolated pancreatic islets for the treatment of diabetes mellitus [1-4]. However, ultimate tissue rejection remains a formidable problem in allogeneic or xenogeneic transplantation. Immunosuppressants have been shown to achieve only minimal success in prolonging the survival of allogeneic islets of Langerhans in rats and man $[1,5]$. Our present approach is focused upon the development of a device which would circumvent the graft rejection problems. The prototype of such a device is an implantable artificial endocrine pancreas (IAEP), consisting of isolated pancreatic islets embedded within a synthetic capillary unit. By virtue of the physical barrier created by the semi-permeable capillary wall excluding molecules above a particular size, host lymphocytes and antibodies are excluded whereas nutrients are allowed to enter the extracapillary space of the unit by diffusion. The islets within the unit are thus isolated from immune destruction and are capable of responding to the physiological needs of the diabetic host.

In a previous communication, we have reported that there was improvement of hyperglycaemia and increase in circulating insulin levels in diabetic rats in acute experiments of $13 \mathrm{~h}$ following implantation of an artificial endocrine pancreas unit [6]. In the present paper, insulin and glucose levels of the diabetic rat following implantation with IAEP containing both islet allograft and xenograft were evaluated for a longer period of up to 11 days during fasting and feeding as well as following an intravenous glucose tolerance test.

\section{Materials and Methods}

Diabetes was induced in inbred male Lewis rats (300-320 g) (Microbiological Associates, Bethesda, Md.) with IV streptozotocin ( $55 \mathrm{mg} / \mathrm{kg}$ body weight). An animal was defined as diabetic only when daily urine output was greater than $100 \mathrm{ml}$ for at least two weeks and when fasting blood glucose level was greater than $400 \mathrm{mg} / \mathrm{dl}$. 


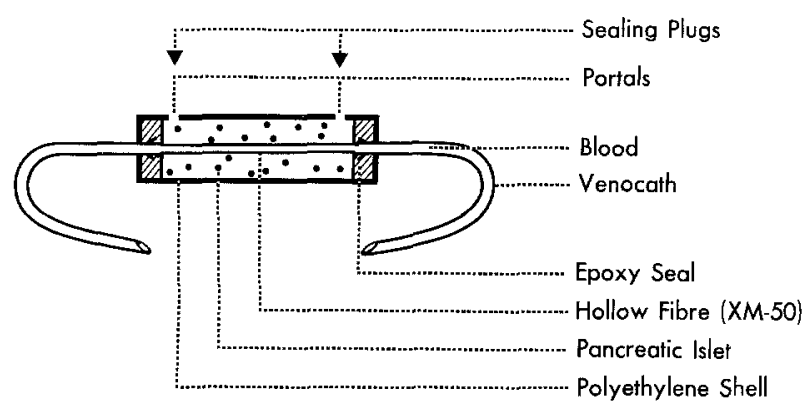

Fig. 1. Diagram to show the construction of the implantable artificial endocrine pancreas

In vivo studies employed an IAEP consisting of functional islets contained within a device which was permeated with a single capillary fibre. These fibres were made of XM-50 acrylic copolymer with a nominal molecular weight cut-off of $50,000,450 \mu \mathrm{m}$ in internal diameter, and a wall thickness of approximately $150 \mu \mathrm{m}$ (Amicon Corp., Lexington, Mass). To make the unit, a $2.8 \mathrm{~cm}$ long fibre was connected by medical grade epoxy resin at both ends with $5 \mathrm{~cm}$ pieces of $18 \mathrm{G}$ bore radiopaque intravenous catheter (Abbott Lab., N Chicago, Ill.). The fibre-catheter was inserted into a $3 \mathrm{~cm}$ long polyethylene shell which was subsequently sealed off at the fibre catheter junction at both ends (Figure 1).

Endocrine pancreatic tissue was isolated from [1] male Lewis $x$ Brown Norway $F_{1}\left(\mathrm{LBNF}_{1}\right)$ hybrid rats weighing approximately $300 \mathrm{~g},[2]$ rabbit and [3] human pancreas by the collagenase digestion method of Lacy and Kostianovsky [7]. Isolated islets were purified from the exocrine debris by Hypaque-Ficoll density centrifugation as previously described by Tze et al. [8]. The technique used for the isolation of human islets was based on the method of Goldman and Colle [9]). The islets were suspended in Medium 199 (Microcan, Calgary, Alberta) which was supplemented with 10 per cent foetal calf serum, $100 \mathrm{U} / \mathrm{ml}$ penicillin and $0.1 \mathrm{mg} / \mathrm{ml}$ streptomycin. The islet suspension was introduced into the IAEP via one of the two portals and the portals were then plugged and sealed. Each artificial capillary unit contained 1,200-1,600 allogeneic rat or approximately 3,000 xenogeneic human or rabbit whole islets. Prior to implantation the artificial endocrine pancreas unit was perfused overnight in a humidified $\mathrm{CO}_{2}$ incubator at $37^{\circ} \mathrm{C}$ with $125 \mathrm{ml}$ of Medium 199 supplemented with 10 per cent foetal calf serum, $100 \mathrm{U} / \mathrm{ml}$ penicillin and $0.1 \mathrm{mg} / \mathrm{ml}$ streptomycin which was circulated at a rate of $1.5-2.0 \mathrm{ml} / \mathrm{min}$ by a Holter pump.

In the in vivo experiments, an $18 \mathrm{G}$ bore radiopaque intravenous catheter was inserted into the jugular vein of the recipient diabetic rat $24 \mathrm{~h}$ prior to the implantation procedure to facilitate the collection of blood samples, the infusion of heparin, and the replacement of isogeneic blood during the ensuing experiment. The catheter was kept open with $0.154 \mathrm{~mol} / 1$ saline containing 10 $\mathrm{U} / \mathrm{ml}$ heparin. The IAEPs containing islets were implanted as a vascular shunt in the abdominal aorta of the rats which have been diabetic for 3-4 weeks. An incision $3 \mathrm{~cm}$ long was made along the linea alba and the abdominal aorta was exposed. Two clamps were applied on the abdominal aorta between the renal and iliolumbar arteries. The implant was subsequently inserted directly into the abdominal aorta. Following the surgical procedure, animals were given water ad libitum, while food was withheld for the first $12-24 \mathrm{~h}$.

A total of 26 diabetic rats received IAEP containing allogeneic islets. Throughout their survival period, plasma glucose and insulin levels were determined. An intravenous glucose tolerance test (IVGTT) was performed on the 3 rd day after implantation in two diabetic rat recipients of the IAEP. This was repeated on the 7 th day in the one animal which survived for 11 days following implantation.

Five diabetic rats received xenogeneic islets. Plasma glucose and insulin levels were determined throughout their survival period after implantation. IVGTT was performed on the 2 nd and 4 th days in one of the recipients of IAEP containing rabbit islets $(n=4)$ and human islets $(n=1)$ respectively.

In all the IAEP recipients, blood samples were taken prior to and at various intervals, from $30 \mathrm{~min}$ to $24 \mathrm{~h}$, following implantation throughout their survival period. Blood samples were also obtained in relation to the feeding and fasting periods in diabetic rat recipients which have survived for more than $24 \mathrm{~h}$. The quantity of blood withdrawn $(0.4 \mathrm{ml}$ per sample) was replaced with isogeneic blood. In order to maintain a free circulation through the implanted unit, the recipient animal received a priming dose of 50 $\mathrm{U}$ of heparin IV during IAEP implantation. An Alzet minipump with a delivery rate of $25 \mathrm{U}$ of heparin/h was implanted intraperitoneally with the catheter connected to the deep circumflex iliac vein to maintain systemic heparinization. The patency of the artificial shunt was determined by means of a Medsonics Ultrasound Stethoscope BF5a. For IVGTT the rats were fasted for 15 hours before the test then glucose $(1 \mathrm{gm} / \mathrm{kg})$ was administered as a $50 \%$ solution through the jugular vein catheter.

Pre- and post-infusion blood samples $(0,5,10,30,45,60,120$ and $180 \mathrm{~min}$ ) were withdrawn for insulin and glucose determination. All plasma glucose levels were measured with the Beckman glucose analyzer. Plasma insulin (IRI) levels were determined by the double antibody immunoassay techniques of Hales and Randle [10].

\section{Results}

Allogeneic Experiments: Figure 2 demonstrates the changes in circulating blood glucose and insulin concentration in 20 diabetic rats which were implanted with the artificial endocrine units containing allogeneic rat islets. Following IAEP implantation, a gradual decrease in plasma glucose and increase in insulin levels were observed and normoglycaemia was achieved within five hours. Plasma insulin increased shortly after transplantation, peaked at $7 \mathrm{~h}$ and subsequently declined as the glucose level stabilized in the normoglycaemic range. In this group of rats survival time varied from $12-24 \mathrm{~h}$. The major cause of the short survival period was mainly due to excessive bleeding from systemic heparinization.

A total of 4 rats survived between 1-3 days. The plasma glucose and insulin levels reached near normal values approximately $5 \mathrm{~h}$ after implantation and continued during the entire survival period of the recipients (Figure 3 ). The profile of insulin and glucose concentration of the two diabetic rats which survived for 4 days and 11 days respectively following the implantation of an IAEP are shown in Figure 4.

Increased circulating levels of insulin and an improvement of the hyperglycaemic state is evident following implantation. As plasma glucose increased with feeding, there was a corresponding increase in the insulin level. This change in the circulating insulin 


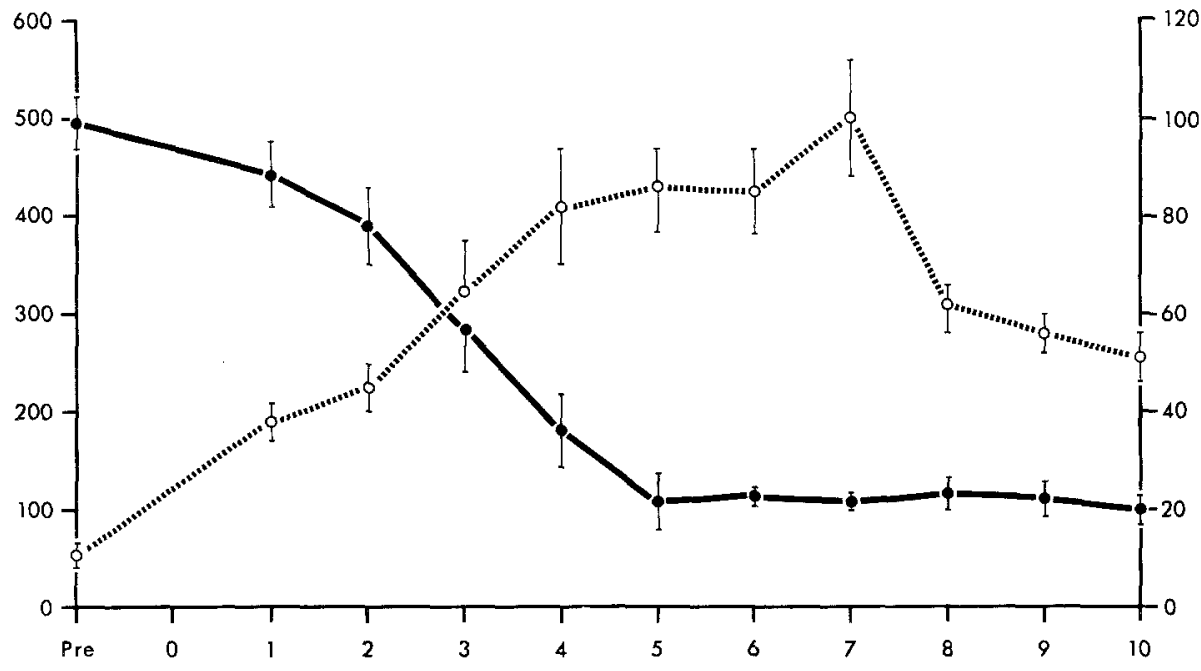

Fig. 2. The plasma glucose and insulin (O-..-O) levels in 20 diabetic rats following implantation of an IAEP containing 1,200 allogeneic rat islets. Values are mean \pm SEM. The left and right ordinates show the concentrations of plasma glucose $(\mathrm{mg} / \mathrm{dl})$ and immunoreactive insulin level $(\mu \mathrm{U} / \mathrm{ml})$ respectively

Time (hours)

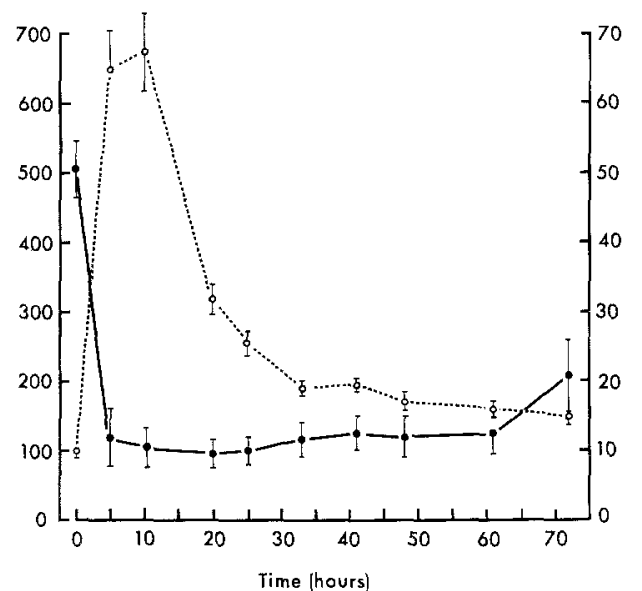

Fig. 3. The plasma glucose (-) and insulin (O-----O) levels in 4 diabetic rats following implantation of an IAEP containing 1,200 allogeneic rat islets. Values are mean \pm SEM. The left and right ordinates show the concentrations of plasma glucose $(\mathrm{mg} / \mathrm{dl})$ and immunoreactive insulin level $(\mu \mathrm{U} / \mathrm{ml})$ respectively

Fig. 4. The plasma glucose (-C) and insulin (०-.---O) levels in 2 diabetic rats which survived 4 and 11 days respectively following the implantation of an IAEP containing 1,200 allogeneic islets under feeding ( $\mathrm{F}$ ) and fasting $(\mathrm{S})$ conditions. The plasma insulin and glucose levels of the IVGTT are shown in Figure 5. The left and right ordinates show the concentrations of plasma glucose $(\mathrm{mg} / \mathrm{dl})$ and immunoreactive insulin level $(\mu \mathrm{U} / \mathrm{ml})$ respectively

level was preceded by changes in the blood glucose level. Figure 5 shows the results of the IVGTT performed on the two recipients of IAEP which survived for 4 and 11 days respectively. Even though the plasma glucose disappearance and insulin release patterns of the IAEP recipients following IVGTT were slower than in normal animals, they were however much improved over the untreated diabetic con-
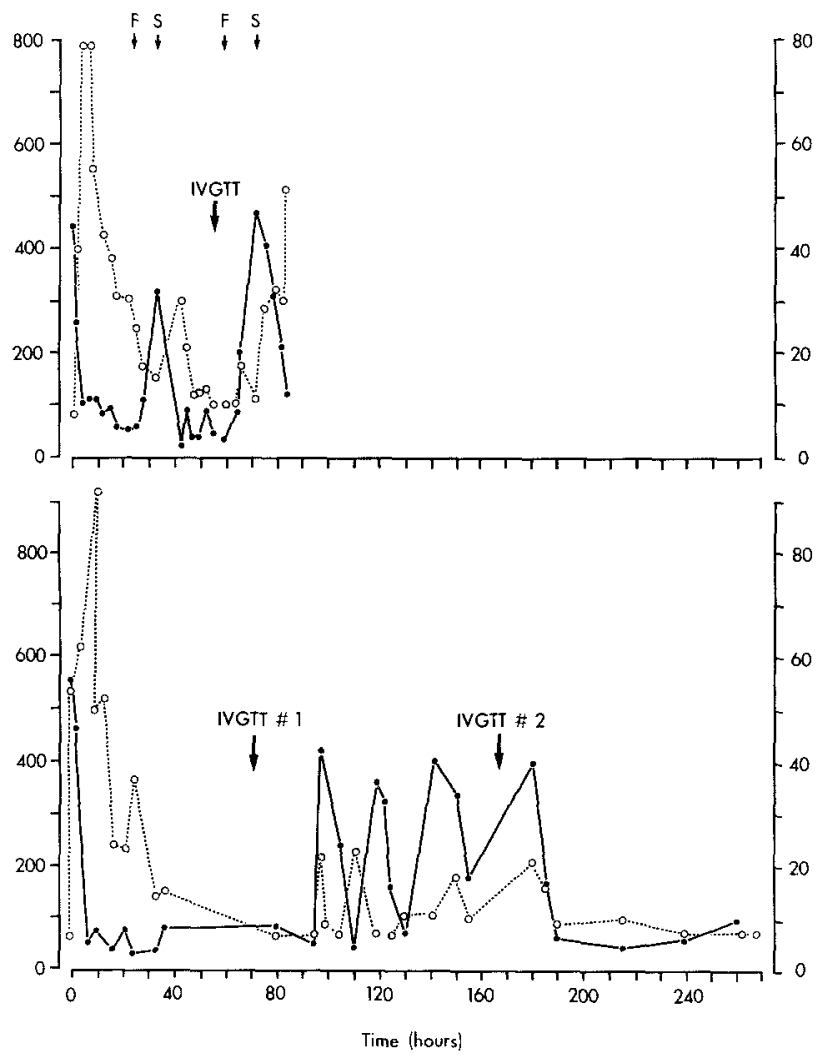

trols undergoing similar test. The implanted device provided the hosts with the capability to respond to the glucose stimulation with the release of insulin resulting in a more normal glucose disappearance pattern.

Xenogeneic Experiments: Figures 6 and 7 reveal the changes in circulating insulin and blood glucose in 

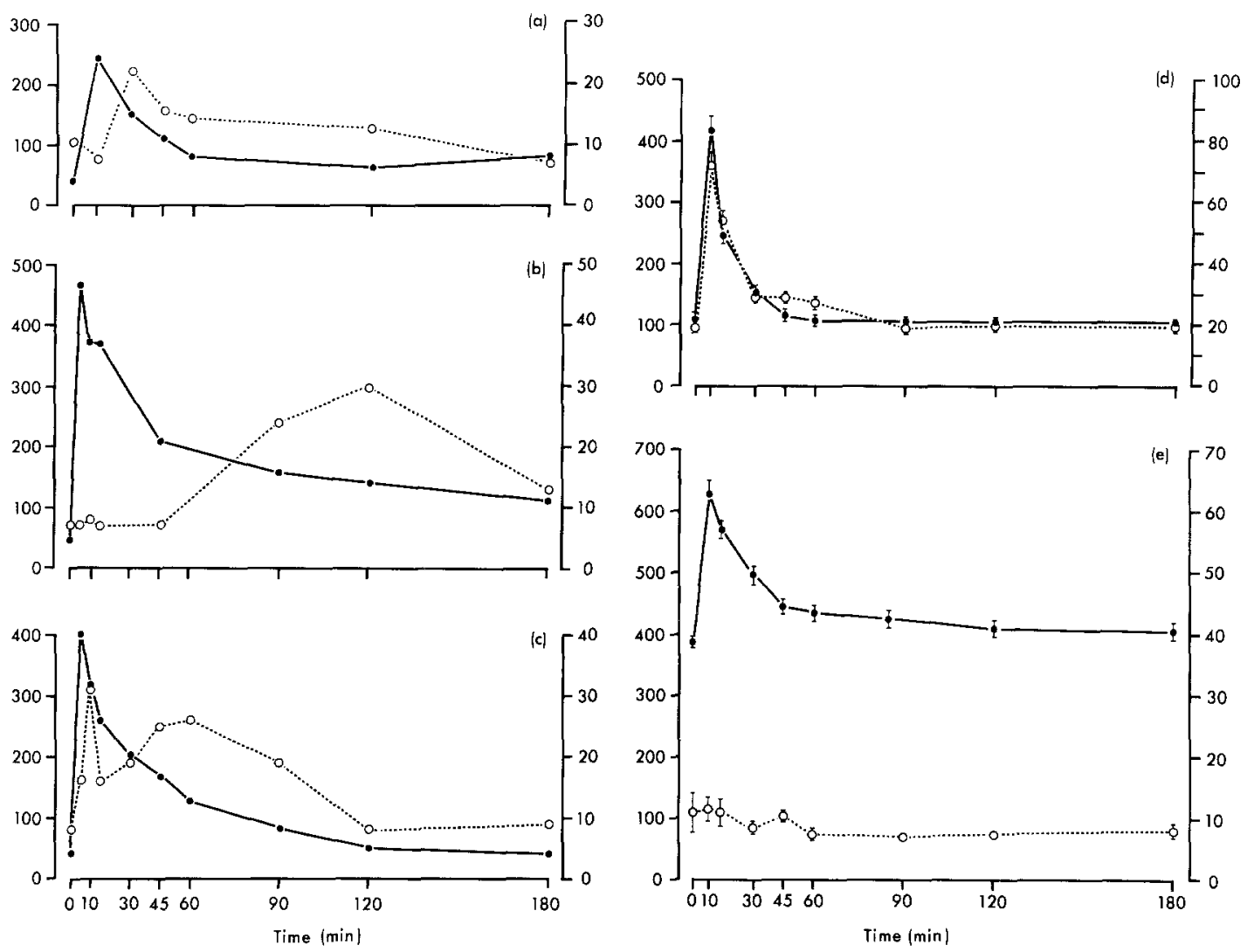

Fig. 5. The plasma glucose (-) and insulin (O----O) levels of the IVGTT performed on 2 diabetic rats which were implanted with an IAEP containing allogeneic islets. The test was performed on day 3 on the rat which survived for 4 days (a) and on days 3 and 7 on the rat which survived for 11 days (b) and (c). Plasma glucose and insulin values, mean \pm SEM, of 4 normal and 4 diabetic rats are shown in (d) and (e) respectively. The left and right ordinates show the concentrations of plasma glucose (mg/dl) and immunoreactive insulin level $(\mu \mathrm{U} / \mathrm{ml})$ respectively

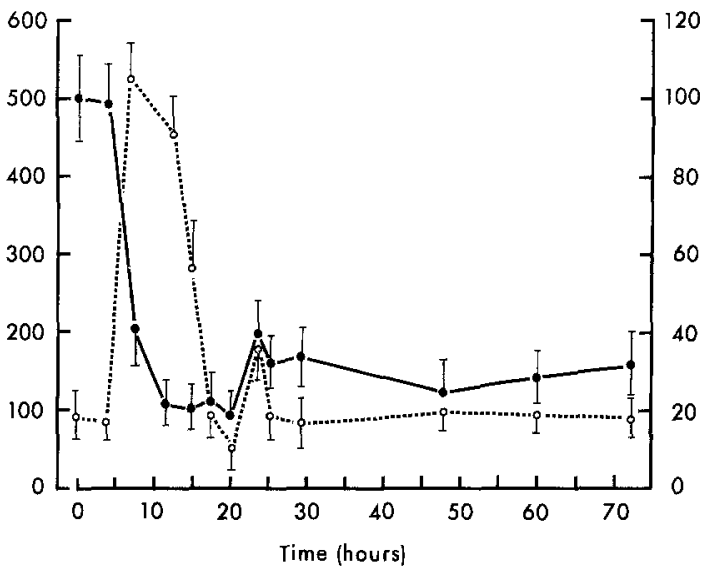

Fig. 6. The plasma glucose (-) and insulin (O----O) levels in 4 diabetic rats following the implantation of IAEP containing approximately 3,000 xenogeneic rabbit islets. The insulin and glucose levels of the IGVTT are shown in Figure 8 (a). Values are mean \pm SEM. The left and right ordinates show the concentrations of plasma glucose $(\mathrm{mg} / \mathrm{dl})$ and immunoreactive insulin level $(\mu \mathrm{U} /$ ml) respectively five diabetic rat recipients which were implanted with IAEPs containing approximately 3,000 xenogeneic rabbit islets $(n=4)$ or human islets $(n=1)$ respectively. Following implantation, a gradual decrease in blood glucose was found but normoglycaemia was not achieved until ten hours later. Figure 8 shows an increase in circulating insulin concentration and an improved glucose disappearance pattern in IVGTT performed on two diabetic rats receiving either rabbit or human xenogeneic islet graft respectively.

\section{Discussion}

In an effort to overcome immunorejection in tissue transplantation, membrane barriers have been used to separate the graft from the host tissue. Pancreatic islets in millipore chambers, when implanted into obese, hyperglycaemic mice, have been demonstrated to ameliorate the hyperglycaemic state in these animals $[11,12]$. However, a major drawback 
with the millipore chamber approach has been the overgrowth of fibroblasts adhering onto the external surface of the chamber which leads to a reduction of their surface porosity for molecular exchange across the membrane. The IAEP system has been devised so that the artificial capillary is enclosed by an outer casing to prevent fibroblast overgrowth on the extracapillary surface. This objective has been achieved in an in vitro study conducted by the authors when islets were cultured in the extracapillary space. The extracapillary surface was free of fibroblasts for the duration of the study for a period of up to 3 months [13]. The additional advantages of the IAEP over millipore chamber is that there is a continuous blood flow which permits quick exchange of nutrients and metabolites between the host's circulating blood and the transplanted islets suspended in the extracapillary space.

A significant time lag in the correction of hyperglycaemia in the diabetic recipients following IAEP implants was observed in our data. The pattern of delay can be explained by the fact that the implantable units used in the in vivo study were constructed with a relatively thick walled large bore artificial capillary fibre. As molecular transport depends greatly on the thickness and surface area of the barrier and volume of the extracapillary space, the delay in achievement of insulin and glucose equilibrium between the extracapillary space and the host circulation would result in a reactive hypoglycaemia. To overcome this problem, units constructed with a larger surface area, reduced extracapillary space and a thinner membrane barrier will theoretically increase the efficiency of molecular exchange across the membrane and result in a decrease of time lag.

The implantation of IAEP as a vascular shunt has an inherent problem of blood clotting; the magnitude of which has been reflected in the early mortality of recipient animals and early termination of experiments, the longest survival period being $48 \mathrm{~h}$ [14-16]. In the present study, although the majority of the animals survived only 1 to 3 days, one of the IAEP recipient rats survived as long as 11 days following implantation. Blood loss from anticoagulation such as intense heparinization schedules, has been the major cause of the short survival period of the experimental animals. To resolve these problems, an improved form of anticoagulation regimen, the application of more nonthrombogenic and biocompatible synthetic materials, and better transport efficiency with optimal membrane thickness would enhance the survival of IAEP recipient diabetic animals.

The potential clinical application of islet transplants has been hindered by two major problems: immunorejection and lack of donor tissue. Pancreatic

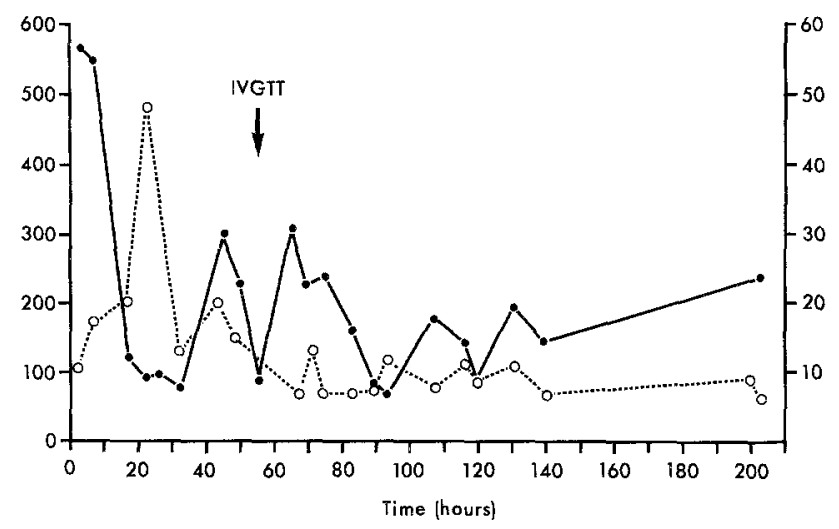

Fig. 7. The plasma glucose (- ) and insulin (O----O) levels in a diabetic rat following the implantation of IAEP containing approximately 3,000 human islets. The insulin and glucose levels of the IVGTT are shown in Figure 8 (b). The left and right ordinates show the concentrations of plasma glucose $(\mathrm{mg} / \mathrm{dl})$ and immunoreactive insulin level $(\mu \mathrm{U} / \mathrm{ml})$ respectively
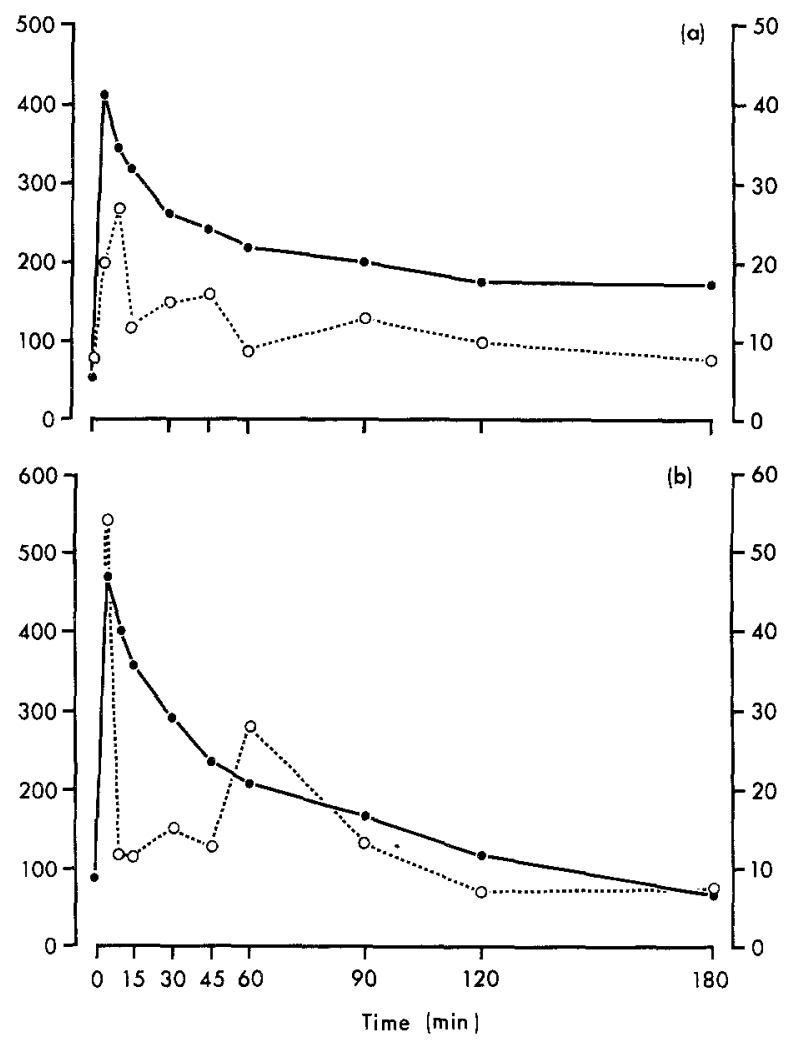

Fig. 8. The plasma glucose (-) and insulin (O----O) levels of the IVGTT performed on 2 diabetic rats which were implanted with an IAEP containing 3,000 xenogeneic islets, rabbit (a) and human (b). The left and right ordinates show the concentrations of plasma glucose $(\mathrm{mg} / \mathrm{dl})$ and immunoreactive insulin level $(\mu \mathrm{U} / \mathrm{ml})$ respectively 
islets have been shown to be highly immunogenic and sensitive to immunorejection effector mechanisms [17]. The imposition of a mechanical barrier between donor tissue and the host has long been proposed as a possible means to circumvent the immunological rejection problem. Immunological studies recently carried out in this laboratory have demonstrated that the allogeneic or xenogeneic graft could be isolated from immunodestruction in a presensitized host by the manipulation of the pore size of the artificial capillary membrane of the IAEP [18]. This finding, combined with the success in achieving amelioration of hyperglycaemia in the diabetic recipient animal with the xenogeneic and allogeneic islet tissue when applied in the IAEP system, would have the potential to resolve the obstacles of islet cell transplantation and pave the way toward making clinical application a reality.

Acknowledgements. This work was supported by grants from the Medical Research Council of Canada, the Heighway Fund and the Canadian Diabetic Association, Vancouver. The authors are grateful to Dr. K. A. Evelyn and Dr. S. Israels for their continuous support and advice, Dr. H. Goldman's help in isolation of human pancreatic islets, Simon O'Young, Joseph Tai and Margaret Tkachuk for excellent technical assistance and to Amicon Corporation for supply of the fibres.

\section{References}

1. Matas, A. J., Sutherland, D. E. R., Najarian, J. S.: Current status of islet and pancreas transplantation in diabetes. Diabetes 25, 785-795 (1976)

2. Maugh, T.H.: Diabetes therapy: Can new techniques halt complications. Science 190, 1281-1284 (1975)

3. Gray, B. N., Watkins, E.: Prevention of vascular complications of diabetes by pancreatic islet transplantation. Arch. Surg. 3, 254-257 (1976)

4. Mauer, S. M., Sutherland, D. E. R., Steffes, M. W., Leonard, R. J., Najarian, J. S., Michael, A. F., Brown, D. M.: Pancreatic transplantation: Effects on the glomerular lesions of experimental diabetes in the rat. Diabetes 23, 748-753 (1974)

5. Marquet, R. L., Heystek, G. A.: The effect of immunosuppressive treatment on the survival of allogeneic islets of Langerhans in rats. Transplantation 20, 428-491 (1975)
6. Tze, W. J., Wong, F. C., Chen, L. M., O'Young, S.: Implantable artificial endocrine pancreas unit used to restore normoglycemia in the diabetic rat. Nature 264, 466-467 (1976)

7. Lacy, P. E., Kostianovsky, M.: Method for the isolation of intact islets of Langerhans from the rat pancreas. Diabetes 16, 35-39 (1967)

8. Tze, W. J., Wong, F. C., Tingle, A. J.: The use of hypaqueficoll in the isolation of pancreatic islets in rats. Transplantation 22, 201-205 (1976)

9. Goldman, H., Colle, E.: Human pancreatic islets in culture: Effects of supplementing the medium with homologous and heterologous serum. Science 192, 1014-1016 (1976)

10. Hales, C.N., Randle, P. J.: Immunoassay of insulin with insulin antibody precipitate. Biochem. J. 88, 137-146 (1963)

11. Strautz, R. L.: Studies of hereditary obese mice (obob) after implantation of pancreatic islets in millipore filter capsules. Diabetologia 6, 106-112 (1970)

12. Gates, R. J., Hunt, M. I., Smith, R., Lazarus, N. F.: Return to normal blood glucose, plasma insulin and weight gain in New Zealand obese mice after implantation of islets of Langerhans. Lancet 1972 II, 567-570

13. Tze, W. J., Chen, L. M.: Long term survival of adult rat islets of Langerhans in artificial capillary culture units. Diabetes $\mathbf{2 6}$, 185-191 (1977)

14. Sun, A. M., Parisius, W., Healy, G. M., Vacek, I., MacMorine, H. G.: The use in diabetic rats and monkeys of artificial capillary units containing cultured islets of Langerhans (artificial endocrine pancreas). Diabetes 26, 1136-1139 (1977)

15. Chick, W. L., Perna, J. J., Lauris, W., Low, D., Galletti, P. M., Panol, G., Whittemore, A.B., Like, A. A., Colton, C. K., Lysaght, M. J.: Artificial pancreas using live beta cells; Effects on glucose homeostasis in diabetic rats. Science 97, 780-782 (1977)

16. Orsetti, A., Guy, C., Zouari, N., Deffay, F.: Implantation du distributeur bio-artificiel d'insuline chez le chien utilizant des îlots de Langerhans d'espèces animales différentes. C. R. Soc. Biol. (Paris) 172, 144-150 (1978)

17. Barker, C. F.: Summary of the 12 th research symposium of the transplantation of islets of Langerhans and the histocompatibility of endocrine tissue. Diabetes 24, 766-775 (1975)

18. Tze, W. J., Wong, F. C., Tai, J.: Immunological isolation of allogeneic or xenogeneic cells in an implantable artificial capillary unit. Transplantation (accepted)

Received: June 20, 1978,

and in revised form: October 9, 1978

Dr. W. J. Tze

Children's Hospital

250 West 59th Avenue

Vancouver, B. C.

V5X 1 X2, Canada 